\title{
A STABLE VARIATIONAL FORMULATION FOR NON-ORDINARY STATE-BASED PERIDYNAMICS
}

\author{
Yuqi Sun ${ }^{1}$,Fengjun $\mathrm{Chen}^{2}$,Xinxin $\mathrm{Wang}^{2}$,Wen Shen ${ }^{2}$,Haitao $\mathrm{Yu}^{3}$ \\ ${ }^{1}$ Department of Geotechnical Engineering, Tongji University, Shanghai, China \\ 200092 \\ 2 Shanghai Construction Group Co., Ltd, Shanghai, China \\ 200080
}

${ }^{3}$ State Key Laboratory of Disaster Reduction in Civil Engineering, Tongji University, Shanghai,
China
200092
yuhaitao@tongji.edu.cn

Key words: Peridynamics, non-ordinary state-based, variational formulation, zero-energy mode control

\begin{abstract}
The paper builds a stable variational formulation for the non-ordinary state-based peridynamics (NOSB-PD). Firstly, a new force state vector is reformulated by introducing the first Piola-Kirchhoff stress in continuum mechanics. The consistency of the new governing equation of the proposed pridynamic model and classical continuum mechanics is proved. Secondly, a stable variational formulation of non-ordinary state based peridynamics is developed to unify the boundary conditions in peridynamcis and continuum mechanics. The zero mode oscillations of non-ordinary state based peridynamics is also eliminated by penalty method in numerical implementation. Numerical examples are illustrated to validate the proposed method. Numerical solutions obtained by the proposed method also indicate that the proposed method can well capture the general nonlinear behavior of solid materials.
\end{abstract}

\section{INTRODUCTION}

Discontinuous problems, such as fracture and collapse, are fundamentally important issues in computational mechanics. One of the critical issues is how to accurately capture the stress field in discontinuities and predict the pattern of the crack. Recently, many significant contributions have been made to simulate the fracture process of solid materials, such as Extended Finite Element Method (XFEM) [1], Element-Free Galerkin (EFG) Method [2] and Material Point Method (MPM) [3].However, these methods either require additional variables and equations to capture cracks, or need complex shape functions to represent displacement and stress fields, which would be difficult for complex fracture problems.

Peridynamics (PD)[4] is a nonlocal reformulation of the classical continuum mechanics (CCM) 
and represents a new theory for the simulation of fracture mechanics. Generally, there are two kinds of peridynamic models are developed, i.e. bond-based and state-based peridynamic models. However, one of the disadvantages of the bond-based PD is that the response of a bond is independent of other bonds, which makes the Poisson's ratio restricted to $1 / 4$ for the plane strain problem and $1 / 3$ for the plane stress problem $[4,5]$. Many significant contributions have been made to solve the limitation of Poisson's ratio in BB-PD, the readers can refer to $[6,7,8,9,10]$. The ordinary state-based (OSB) PD and the non-ordinary state-based (NOSB) $\mathrm{PD}$ overcomes the defects of BB-PD. One important feature of NOSB-PD is that it incorporates constitutive model from the CCM such as plasticity [11] and visco-plasticity [12] into the force state. However, the zero-energy mode oscillation induced by the "PD correspondence material models"[13] in NOSB-PD is still a challenging issue in peridynamic community.

Generally, the simulation of PD is based on the strong form of balance equation of linear momentum and has difficulties in imposing boundary conditions, and thus may result in truncated errors in the horizon of a material point near the boundary surface.

This paper aims to build a stable variational formulation for peridynamics. Firstly, the first Piola-Kirchhoff stress is introduced to reformulate the force state vector in original non-ordinary state based peridynamics. This new force state vector ensures that the strong form of PD is theoretically consistent with that of CCM when the nonlocal effects of material points are ignored. Secondly, a stable variational formulation is proposed to unifies the boundary conditions of PD and CCM, which makes the proposed method easier work with various boundary conditions

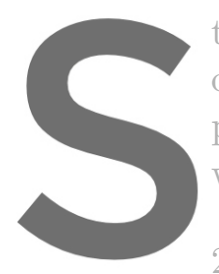
than standard peridy namics. Moreover, a pen
oscillation of NSOB-PD and ensure the computa
presented to demonstrate the validation of the
with those of analytical solutions and experime 2 The strong form and variational framework
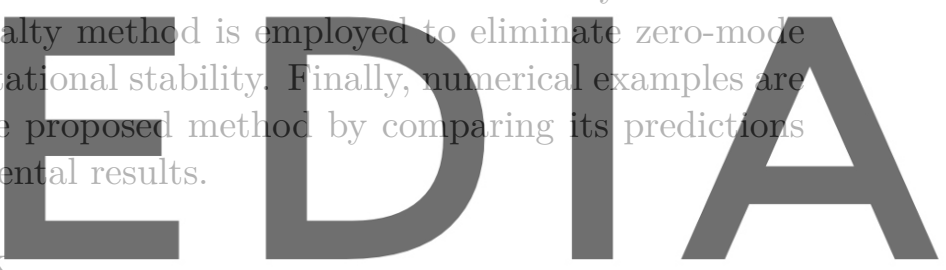

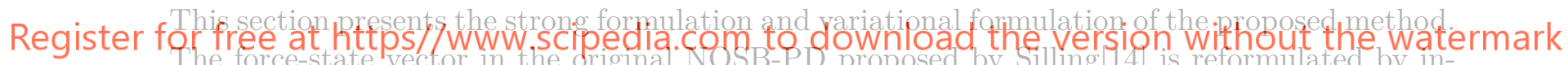
troducing the first Piola-Kichhoff stress.

\subsection{The strong form}

The linear momentum equation of the proposed model in the referential configuration $\Omega_{o}$ can be given by

$$
\rho_{o} \frac{\partial^{2} \mathbf{U}(\mathbf{X}, t)}{\partial t^{2}}=\int_{H_{\mathbf{X}}}\left(\underline{\mathbf{T}}\left[\mathbf{X}^{\prime}, t\right]\left\langle\mathbf{X}^{\prime}-\mathbf{X}\right\rangle+\underline{\mathbf{T}}[\mathbf{X}, t]\left\langle\mathbf{X}-\mathbf{X}^{\prime}\right\rangle\right) d V_{\mathbf{X}^{\prime}}+\mathbf{B}(\mathbf{X}, t)
$$

where $\rho_{o}$ is the mass density in the referential configuration $\Omega_{o}, \mathbf{U}(\mathbf{X}, t)$ is the displacement of material point $\mathbf{X}$ at time $t, H_{\mathbf{X}}$ is the support domain of matrial point $\mathbf{X}, \mathbf{T}[\mathbf{X}, t]\left\langle\mathbf{X}-\mathbf{X}^{\prime}\right\rangle$ and $\underline{\mathbf{T}}\left[\mathbf{X}^{\prime}, t\right]\left\langle\mathbf{X}^{\prime}-\mathbf{X}\right\rangle$ are the force state vectors of material points $\mathbf{X}$ and $\mathbf{X}^{\prime}$, respectively, $d V_{\mathbf{X}^{\prime}}$ is the volume of material point $\mathbf{X}^{\prime}$ in $H_{\mathbf{X}}$ and $\mathbf{B}(\mathbf{X})$ is the external body force density of material point $\mathbf{X}$. The angle brackets in the force state $\underline{\mathbf{T}}[\mathbf{X}, t]\left\langle\mathbf{X}-\mathbf{X}^{\prime}\right\rangle$ denotes this force state vector operates on bond $\mathbf{X}-\mathbf{X}^{\prime}$. By introducing the first Piola-Kirchhoff stress tensor $\mathbf{P}(\mathbf{X}, t)$ in the CCM, the 
force state defined in Eq.(1) can be reformulated as

$$
\begin{aligned}
& \underline{\mathbf{T}}\left[\mathbf{X}^{\prime}, t\right]\left\langle\mathbf{X}^{\prime}-\mathbf{X}\right\rangle=\omega\left(\left\|\mathbf{X}^{\prime}-\mathbf{X}\right\|\right)\left(\left(\mathbf{X}^{\prime}-\mathbf{X}\right) \otimes \mathbf{P}\left(\mathbf{X}^{\prime}, t\right) \cdot \mathbf{K}^{-1}(\mathbf{X})\right): \mathbf{I}^{(2)} \\
& \underline{\mathbf{T}}[\mathbf{X}, t]\left\langle\mathbf{X}-\mathbf{X}^{\prime}\right\rangle=\omega\left(\left\|\mathbf{X}-\mathbf{X}^{\prime}\right\|\right)\left(\left(\mathbf{X}-\mathbf{X}^{\prime}\right) \otimes \mathbf{P}(\mathbf{X}, t) \cdot \mathbf{K}^{-1}(\mathbf{X})\right): \mathbf{I}^{(2)}
\end{aligned}
$$

where $\boldsymbol{\omega}\left(\left\|\mathbf{X}-\mathbf{X}^{\prime}\right\|\right)$ and $\boldsymbol{\omega}\left(\left\|\mathbf{X}^{\prime}-\mathbf{X}\right\|\right)$ are the influence functions that depend on the length of bond $\mathbf{X}-\mathbf{X}^{\prime}, \mathbf{I}^{(2)}$ is the second order identity tensor, and $\mathbf{K}(\mathbf{X})$ is the shape tensor of material point $\mathbf{X}$, defined by

$$
\mathbf{K}(\mathbf{X})=\int_{H_{\mathbf{X}}} \omega\left(\left\|\mathbf{X}-\mathbf{X}^{\prime}\right\|\right)\left(\mathbf{X}^{\prime}-\mathbf{X}\right) \otimes\left(\mathbf{X}^{\prime}-\mathbf{X}\right) d V_{\mathbf{X}^{\prime}}
$$

Note that the stress tensor field $\mathbf{P}\left(\mathbf{X}^{\prime}, t\right)$ of the neighbor material point $\mathbf{X}^{\prime}$ in $H_{\mathbf{X}}$ can be obtained, using the Taylor series expansion, by

$$
\mathbf{P}\left(\mathbf{X}^{\prime}, t\right)=\mathbf{P}(\mathbf{X}, t)+\nabla_{\mathbf{X}} \mathbf{P}(\mathbf{X}, t) \cdot\left(\mathbf{X}^{\prime}-\mathbf{X}\right)+r\left(\left\|\mathbf{X}-\mathbf{X}^{\prime}\right\|^{2}\right)
$$

where $\nabla_{\mathbf{X}} \mathbf{P}(\mathbf{X}, t)$ denotes the material graident of $\mathbf{P}(\mathbf{X}, t)$ at material point $\mathbf{X}$ in the referential configuration $\Omega_{o}$ and $r\left(\left\|\mathbf{X}-\mathbf{X}^{\prime}\right\|^{2}\right)$ represents the second- and higher-order terms. Substituting Eqs.(2)-(4) into Eq.(1), gives
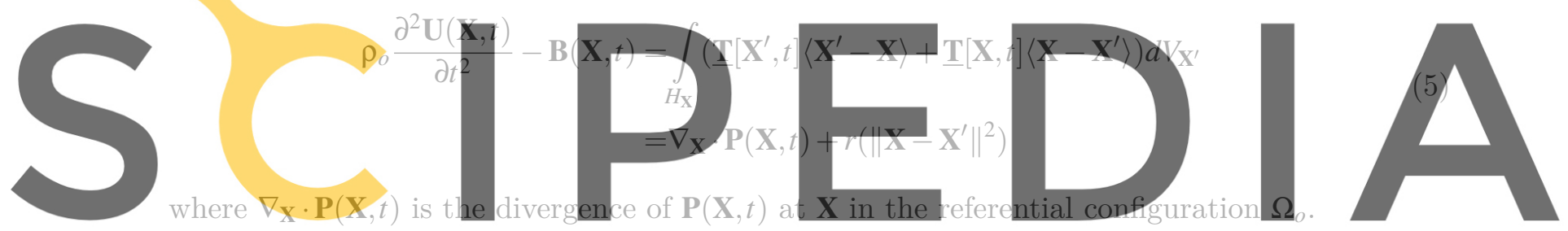

In order to describe the deformation of the continuum and evaluate the stress tensor field, the

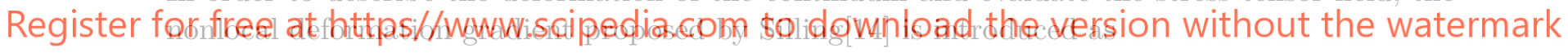

$$
\underline{\mathbf{F}}(\mathbf{X}, t)=\int_{H_{\mathbf{X}}} \omega\left(\left\|\mathbf{X}^{\prime}-\mathbf{X}\right\|\right)\left(\mathbf{U}\left(\mathbf{X}^{\prime}, t\right)-\mathbf{U}(\mathbf{X}, t)\right) \otimes\left(\mathbf{X}^{\prime}-\mathbf{X}\right) d V_{\mathbf{X}^{\prime}} \cdot \mathbf{K}^{-1}(\mathbf{X})+\mathbf{I}^{(2)}
$$

where $\mathbf{U}(\mathbf{X}, t)$ and $\mathbf{U}\left(\mathbf{X}^{\prime}, t\right)$ are the displacements of material points $\mathbf{X}$ and $\mathbf{X}^{\prime}$ in the referential configuration $\Omega_{o}$, respectively.

\subsection{The variational formulation}

In order to obtain the unified variational framework between NOSB-PD and CCM, we begin with the weak form. Each side of Eq.(1) is multiplied by a test funtion $\delta \mathbf{U}(\mathbf{X}, t)$ and is integrated over the domain $\Omega_{o}$, gives

$$
\int_{\Omega_{o}} \delta \mathbf{U}(\mathbf{X}, t) \rho_{o} \frac{\partial^{2} \mathbf{U}(\mathbf{X}, t)}{\partial t^{2}} d V_{\mathbf{X}}=\int_{\Omega_{o}} \delta \mathbf{U}(\mathbf{X}, t) \cdot\left(\nabla_{\mathbf{X}}^{N} \cdot \mathbf{P}(\mathbf{X}, t)\right) d V_{\mathbf{X}}+\int_{\Omega_{o}} \delta \mathbf{U}(\mathbf{X}, t) \mathbf{B}(\mathbf{X}, t) d V_{\mathbf{X}}
$$




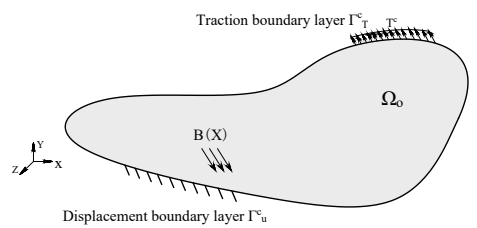

(a)

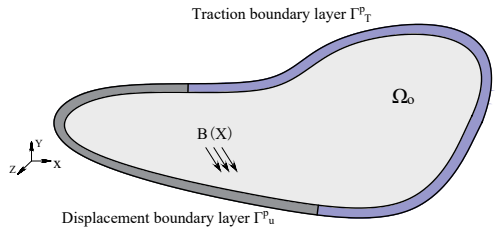

(b)

Figure 1: Displacement and traction boundary conditions in (a) the CCM; and (b) the PD.

where $\nabla_{\mathbf{X}}^{N}$ represents the nonlocal Nabla operator in the VUPD and $\nabla_{\mathbf{X}}^{N} \cdot \mathbf{P}(\mathbf{X}, t)$ is the nonlocal divergence of the first Piola-Kirchhoff stress tensor, defined by

$$
\nabla_{\mathbf{X}}^{N} \cdot \mathbf{P}(\mathbf{X}, t)=\int_{H_{\mathbf{X}}} \omega\left(\left\|\mathbf{X}-\mathbf{X}^{\prime}\right\|\right)\left(\left(\mathbf{X}^{\prime}-\mathbf{X}\right) \otimes \Delta \mathbf{P}\left(\mathbf{X}^{\prime}-\mathbf{X}, t\right) \cdot \mathbf{K}^{-1}(\mathbf{X})\right): \mathbf{I}^{(2)} d V_{\mathbf{X}^{\prime}}
$$

where $\Delta \mathbf{P}\left(\mathbf{X}^{\prime}-\mathbf{X}, t\right)=\mathbf{P}\left(\mathbf{X}^{\prime}, t\right)-\mathbf{P}(\mathbf{X}, t)$.

Fig.1 shows the displacement and traction boundary conditions in the CCM and in the PD. Similar to the test function space in the CCM, the test function $\delta \mathbf{U}(\mathbf{X}, t)$ satisfies

$$
\delta \mathbf{U}(\mathbf{X}, t) \in\left\{\mathbf{v}(\mathbf{X}, t) \mid \mathbf{v}(\mathbf{X}, t) \in C^{o}\left(\Omega_{0} \times[0, T]\right), \mathbf{v}(\mathbf{X}, t)=0 \text { on } \Gamma_{\mathrm{u}}^{p}\right\}
$$

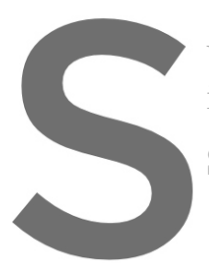

where $\mathbf{v}(\mathbf{X}, t)$ is the ar in Fig.1).

Similarly, a nonlocal
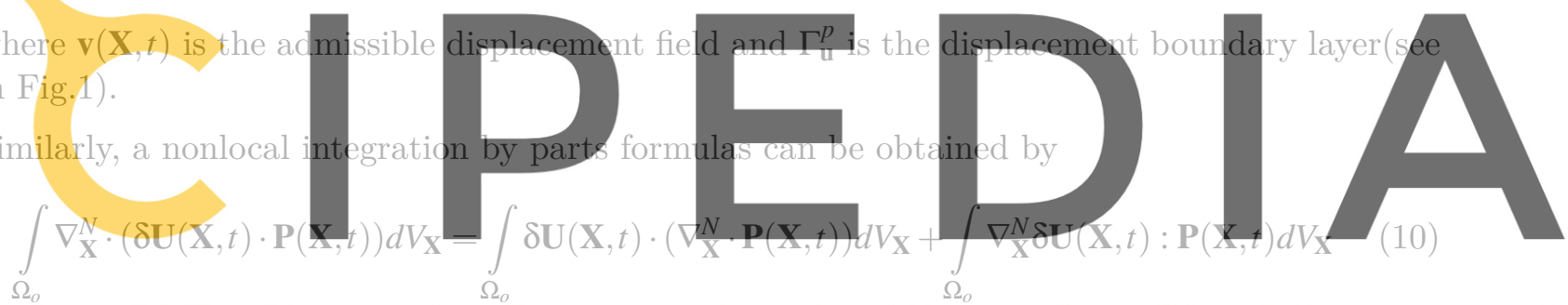

Register for free at https//www.scipedia.com to download the version without the watermark where $\nabla_{\mathbf{X}}^{N} \delta \mathbf{U}(\mathbf{X}, t)$ is the nonlocal gradient of $\delta \mathbf{U}(\mathbf{X}, t)$, which is defined by

$$
\nabla_{\mathbf{X}}^{N} \delta \mathbf{U}(\mathbf{X}, t)=\int_{H_{\mathbf{X}}} \omega\left(\left\|\mathbf{X}-\mathbf{X}^{\prime}\right\|\right)\left(\delta \mathbf{U}\left(\mathbf{X}^{\prime}, t\right)-\delta \mathbf{U}(\mathbf{X}, t)\right) \otimes\left(\mathbf{X}^{\prime}-\mathbf{X}\right) d V_{\mathbf{X}^{\prime}} \cdot \mathbf{K}^{-1}(\mathbf{X})
$$

Since displacement and traction boundary conditions are treated as a fictitious material layer in PD, as seen in Fig.1, the following applies

$$
\int_{\Gamma_{\mathbf{T}}^{c}} \mathbf{P}(\mathbf{X}, t) \cdot \mathbf{N} d S=\int_{\Gamma_{\mathbf{T}}^{c}} \mathbf{T}^{c}(\mathbf{X}, t) d S=\int_{\Gamma_{\mathbf{T}}^{p}} \mathbf{T}^{p}(\mathbf{X}, t) d V_{\mathbf{X}}
$$

where $\Gamma_{\mathbf{T}}^{c}$ is the traction boundary of CCM in the referential configuration $\Omega_{o}$, $\mathbf{T}^{c}$ is the traction exerted on $\Gamma_{\mathbf{T}}^{c}, \mathbf{N}$ is the outer normal of the continuum in $\Omega_{o}, d S$ is the differential area of $\Gamma_{\mathbf{T}}^{c}$, $\Gamma_{\mathbf{T}}^{p}$ represents the fictious traction boundary layer and $\mathbf{T}^{p}$ is the traction exerted on $\Gamma_{\mathbf{T}}^{p}$ in the referential configuration $\Omega_{o}$. 
Using the classical Gauss's theorem, one obtains

$$
\int_{\Omega_{o}} \nabla_{\mathbf{X}}^{N} \cdot(\delta \mathbf{U}(\mathbf{X}, t) \cdot \mathbf{P}(\mathbf{X}, t)) d V_{\mathbf{X}}=\int_{\Gamma_{\mathbf{T}}^{p}} \mathbf{T}^{p}(\mathbf{X}, t) \cdot \delta \mathbf{U}(\mathbf{X}, t) d V_{\mathbf{X}}
$$

Substituting Eq.(13) into Eq.(10), we have

$$
\int_{\Gamma_{\mathbf{T}}^{p}} \mathbf{T}^{p}(\mathbf{X}) \cdot \delta \mathbf{U}(\mathbf{X}, t) d V_{\mathbf{X}}=\int_{\Omega_{o}} \delta \mathbf{U}(\mathbf{X}, t) \cdot\left(\nabla_{\mathbf{X}}^{N} \cdot \mathbf{P}(\mathbf{X}, t)\right) d V_{\mathbf{X}}+\int_{\Omega_{o}} \nabla_{\mathbf{X}}^{N} \delta \mathbf{U}(\mathbf{X}, t): \mathbf{P}(\mathbf{X}, t) d V_{\mathbf{X}}
$$

Combing Eq.(14) and Eq.(7), the weak form can be obtained by

$$
\begin{aligned}
& \int_{\Omega_{o}} \delta \mathbf{U}(\mathbf{X}, t) \rho_{o} \frac{\partial^{2} \mathbf{U}(\mathbf{X}, t)}{\partial t^{2}} d V_{\mathbf{X}}+\int_{\Omega_{o}} \nabla_{\mathbf{X}}^{N} \delta \mathbf{U}(\mathbf{X}, t): \mathbf{P}(\mathbf{X}, t) d V_{\mathbf{X}} \\
& =\int_{\Gamma_{\mathrm{T}}^{p}} \delta \mathbf{U}(\mathbf{X}, t) \cdot \mathbb{T}^{p}(\mathbf{X}, t) d V_{\mathbf{X}}+\int_{\Omega_{o}} \delta \mathbf{U}(\mathbf{X}, t) \cdot \mathbb{B}(\mathbf{X}, t) d V_{\mathbf{X}}
\end{aligned}
$$

Note that the weak form of Eq.(15) is a total Lagrangian formulation in the referential configuration $\Omega_{0}$.
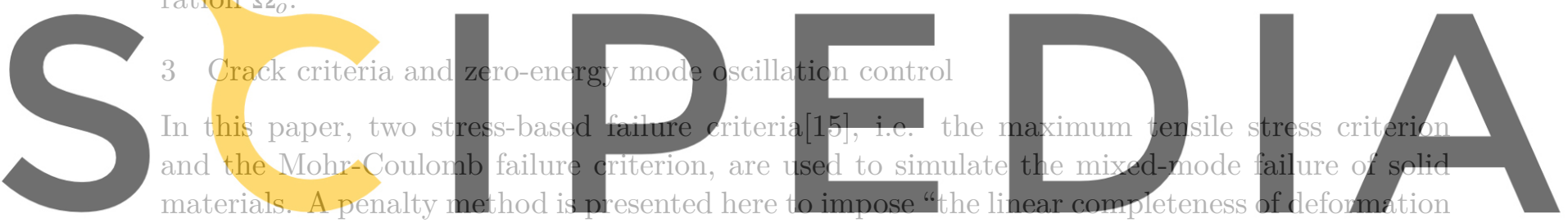

field" in the support domain of each material point, and to obtain the first and second variations

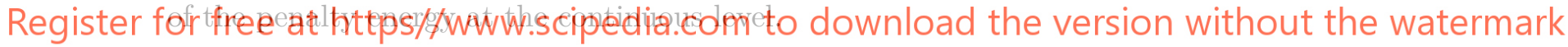

\subsection{Crack criteria}

If we define the Cauchy stresses at two material points $\mathbf{X}$ and $\mathbf{X}^{\prime}$ as $\sigma(\mathbf{X}, t)$ and $\sigma\left(\mathbf{X}^{\prime}, t\right)$, the stress state of bond $\mathbf{X}-\mathbf{X}^{\prime}$ is obtained by

$$
\sigma_{\mathbf{X}-\mathbf{X}^{\prime}}=\frac{1}{2}\left(\sigma(\mathbf{X}, t)+\sigma\left(\mathbf{X}^{\prime}, t\right)\right)
$$

Regarding the first strength criterion for brittle materials, cracking is expected when the maximum tensile stress of the bond reaches the uniaxial tensile strength, i.e.

$$
\sigma_{\mathbf{X}-\mathbf{X}^{\prime}}^{1}>\sigma_{c}
$$

where $\sigma_{\mathbf{X}-\mathbf{X}^{\prime}}^{1}$ is the maximum principal stress of bond $\mathbf{X}-\mathbf{X}^{\prime}$ and $\sigma_{c}$ is the uniaxial tensile strength of brittle materials. 


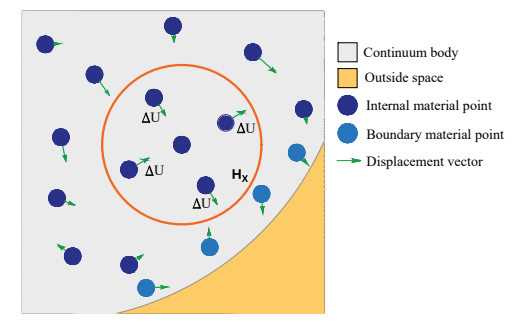

Figure 2: Zero-energy mode in the NSOB-PD.

Similarly, the Mohr-Coulomb failure criterion is employed to describe the shear failure of brittle materials. The shear failure criterion can be expressed by

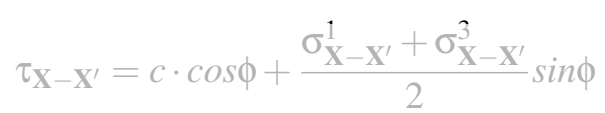

where $\tau_{\mathbf{X}}-\mathbf{X}^{\prime}=\frac{1}{2}\left(\sigma_{\mathbf{X}-\mathbf{X}^{\prime}}^{1}-\sigma_{\mathbf{X}-\mathbf{X}^{\prime}}^{3}\right)$ is the shear stress strength of bond $\mathbf{X}-\mathbf{X}^{\prime}, \sigma_{\mathbf{X}-\mathbf{X}^{\prime}}^{3}$ is the minimum principal stress of bond $\mathbf{X}-\mathbf{X}^{\prime}, c$ is the cohesive strength and $\phi$ is the angle of internal friction.

A local damage parameter $D(\mathbf{X}, t)$ is introduced to describe the damage evolution of materials, which is defined by the ratio of the number of broken bonds to the total number of initial bonds,
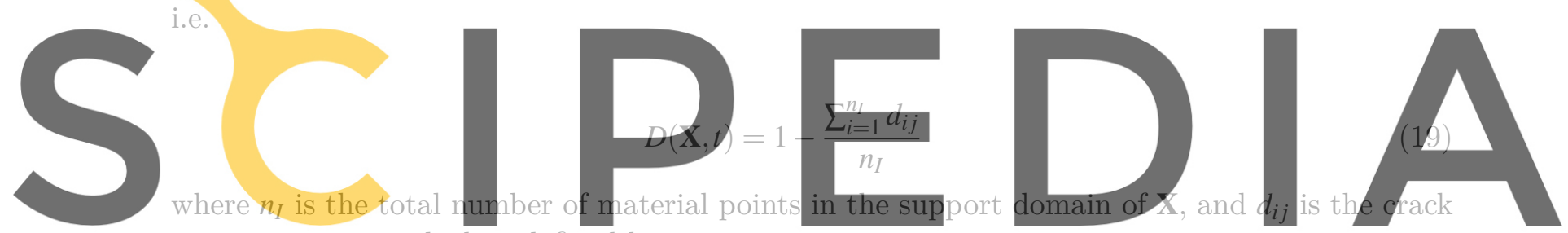

state parameter, which is defined by

Register for free at https//www.scipedia.dom to dowinload the version without the watermark

3.2 Control method for the zero-energy mode oscillation

Fig.2 demonstrates the zero-energy mode in the NOSB-PD. The deformation gradient of the central material point gets $F(\mathbf{X}, t)=\mathbf{I}^{(2)}$ when the family members of this material point have the same displacement $\Delta \mathbf{U}$. This results in the zero-energy mode oscillation in numerical implementation. In this paper, the penalty method is used to eliminate the zero-energy mode oscillation. The non-uniform part of deformation can be defined by as[13]

$$
\mathbf{z}\left(\mathbf{X}^{\prime}, \mathbf{X}, t\right)=\nabla_{\mathbf{X}}^{N} \mathbf{U}(\mathbf{X}, t) \cdot \Delta \mathbf{X}-\Delta \mathbf{U}\left(\mathbf{X}^{\prime}, \mathbf{X}, t\right)
$$

where $\Delta \mathbf{X}=\mathbf{X}^{\prime}-\mathbf{X}$ and $\Delta \mathbf{U}\left(\mathbf{X}^{\prime}, \mathbf{X}, t\right)=\mathbf{U}\left(\mathbf{X}^{\prime}, t\right)-\mathbf{U}(\mathbf{X}, t)$. The penalty potential energy is given by

$$
W_{p}(t)=\int_{\Omega_{o}} \frac{\beta(\mathbf{X})}{2} \int_{H_{\mathbf{X}}} \omega\left(\left\|\mathbf{X}^{\prime}-\mathbf{X}\right\|\right) \mathbf{z}\left(\mathbf{X}^{\prime}, \mathbf{X}, t\right)^{T} \mathbf{z}\left(\mathbf{X}^{\prime}, \mathbf{X}, t\right) d V_{\mathbf{X}^{\prime}} d V_{\mathbf{X}}
$$


Table 1: Geometrical dimensions and material parameters of the double-edge-notched specimen

\begin{tabular}{ll}
\hline Parameters & Values \\
\hline Length(L) & $200 \mathrm{~cm}$ \\
\hline Width(W) $(\mathrm{W})$ & $200 \mathrm{~cm}$ \\
\hline Thickness $(\mathrm{t})$ & $2 \mathrm{~cm}$ \\
\hline Young's modulus(E) & $32 \mathrm{Gpa}$ \\
\hline Poisson's ratio( $(\mathrm{v})$ & 0.2 \\
\hline Axial tensile strength $\left(f_{t}\right)$ & $3 \mathrm{Mpa}$ \\
\hline Axial compressive $\operatorname{strength}\left(f_{t}\right)$ & $38.4 \mathrm{Mpa}$ \\
\hline
\end{tabular}

where $\beta(\mathbf{X})$ is the regularized penalty potential energy coefficient, expressed by

$$
\beta(\mathbf{X})=\frac{\alpha}{\sqrt{\sum_{i=1}^{n_{d}} \lambda_{i}^{2}(\mathbf{X})}}
$$

where $\alpha$ is the penalty potential coefficient related to material point $\mathbf{X}, \lambda_{i}(\mathbf{X})$ is the eigenvalue of the shape tensor $\mathbf{K}(\mathbf{X})$ and $n_{d}$ is the dimension of the space.

4 Numerical examples

Two numerical examples are performed to validate the proposed model by comparing its predictions with those simplicity, the accelerat the Gauss's kernel fuutetion. 4.1 Mixed-mode failure of Double-edge-notch
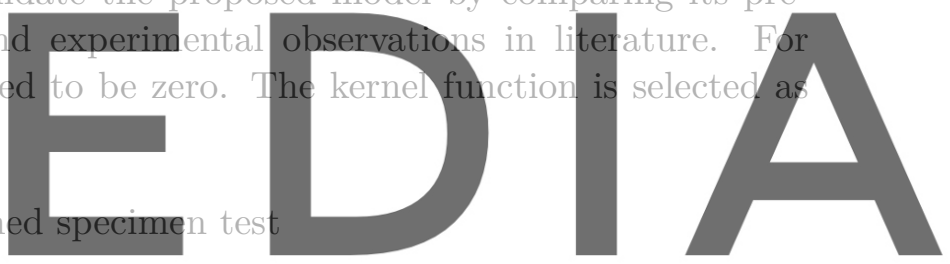

A double-edge-notched specimen under combined shear and tension loads is investigated to

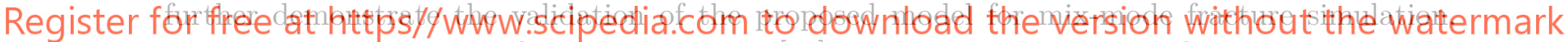

The experimental results from the literature [16] is taken as a benchmark for comparison. The

bottom and right sides of the specimen is fixed, and the tractions are applied on the top and left

sides of the specimen, as shown in Fig.3. According to the experimental data from the literature [16], the geometrical dimensions and material parameters are listed in Table 1.

The specimen is discretized by material points with a uniform spacing $\Delta X=\Delta Y=1 \mathrm{~cm}$. The total number of material points are $N_{p}=38000$, and the penalty potential coefficient $\alpha$ is selected as $5 \mathrm{E}$ in the simulation. The horizontal and vertical displacements with the same increment of $\Delta U=\Delta U_{s}=5 \times 10^{-6}$ for each load step are applied on the left and top sides of the specimen, as shown in Fig.3(a), which is consistent with the "Load Path 2" in [16]. The Mohr-Column failure criterion described in Section 3.1 is adopted in this simulation to predict failure behaviors of the specimen.

Fig.4 shows the predicted crack propagation in the specimen at different load steps, from the proposed model and the experimental observation in literature[16]. The local damage variable $D(\mathbf{X}, t)$ in Eq.(19) is used to describe the failure process of the specimen. Fig.4 also indicates that the crack trajectories predicted by the proposed model agree well with experimental observations 


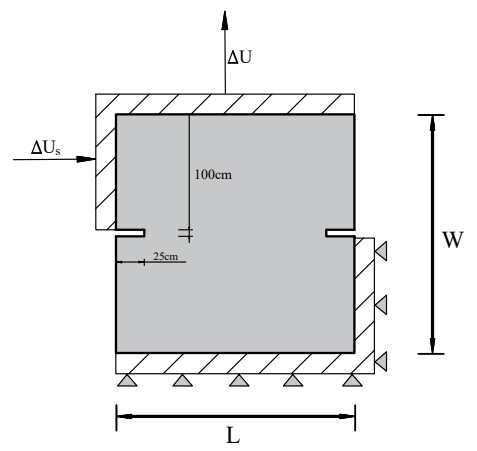

Figure 3: Double-edge-notched specimen under tension-shear combined loads.

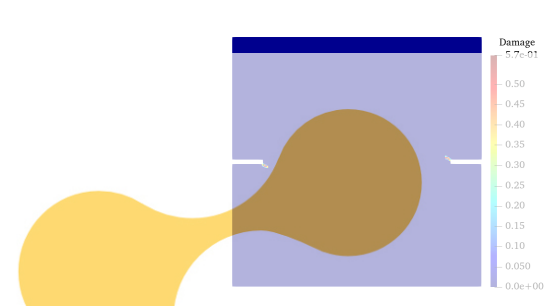

(a)

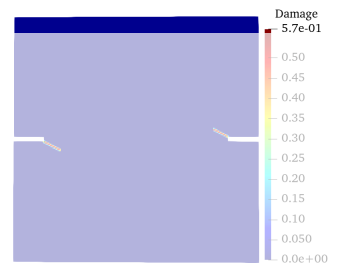

(b)

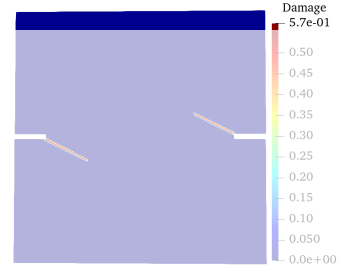

(c)

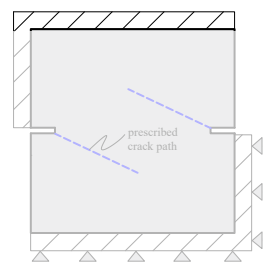

(d)

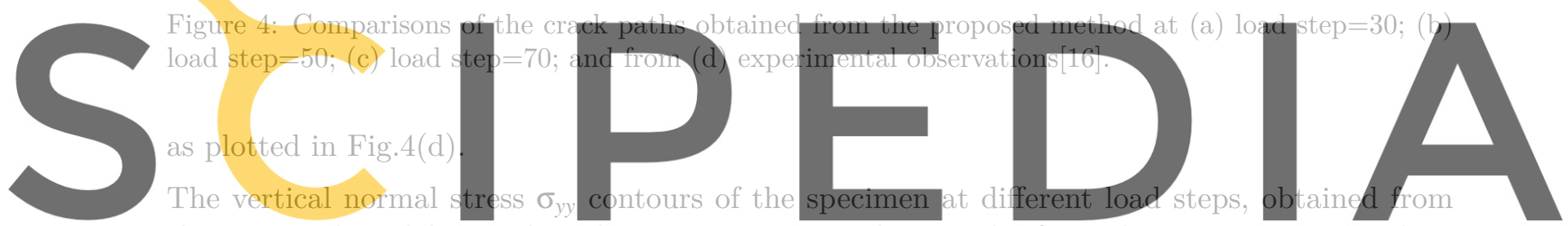

the proposed model, are plotted in Fig.5, respectively. Results from the stress responses show case of mixed-node rracture simulation.

\subsection{Uniaxial tension of a hyper-elastic beam}

To further validate the propos model for large-deformation problems, a beam made of Sant VentKirchhoff hyper-elastic material under uniaxial tension is simulated by the proposed model and then compared with the FEM results. The left end of the hyper-elastic beam is fixed and the traction is applied on the right end of the beam, as shown in Fig.6. The geometrical dimensions and material parameters are listed in Table 2. The total elongation of the hyper-elastic beam is set as $\Delta \mathbf{U}=1 \mathrm{~m}$, and the increment of displacement is specified as $0.01 \mathrm{~m}$ for each load step. The penalty potential energy factor $\alpha$ is selected as $5 \mathrm{E}$ in the simulation.

Four types of discretization with uniform and non-uniform distribution of material points are considered in the simulation, i.e. Cases 1-4. In the uniform case (Case 1), the horizontal and vertical spacings between each two adjacent material points are selected as $\Delta X=0.033$ and $\Delta Y=0.0055$, respectively, and the total number of material points in the model is $N_{p}=5400$. The area of each material point is specified as $A_{I}=\frac{L W}{N p}$. For Cases 2-4, the total number of 


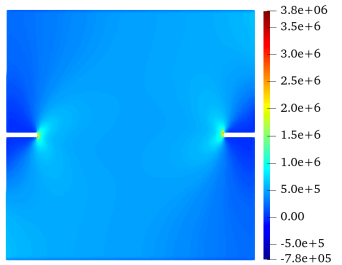

(a)

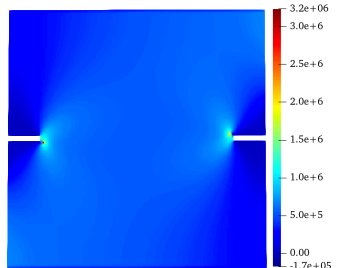

(b)

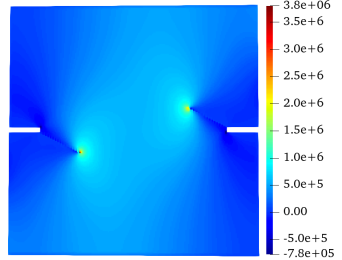

(c)

Figure 5: The stress contour of $\sigma_{y y}$ obtained by the proposed model at (a) load step=30; (b) load step=50; and (c) load step=70 (unit: $\mathrm{Pa}$ ).

Table 2: Geometrical dimensions and material parameters of the hyper-elastic beam

\begin{tabular}{ll}
\hline Parameters & Values \\
\hline Length $(\mathrm{L})$ & $1 \mathrm{~m}$ \\
\hline Cross-sectional area $(\mathrm{A})$ & $0.04 \mathrm{~m}^{2}$ \\
\hline Width $(\mathrm{W})$ & $0.2 \mathrm{~m}$ \\
\hline Young's modulus $(\mathrm{E})$ & $70 \mathrm{GPa}$ \\
\hline Poisson's ratio $(\mathrm{v})$ & 0.33 \\
\hline
\end{tabular}

material points and the area of each material point are the same with Case 1. Note that the

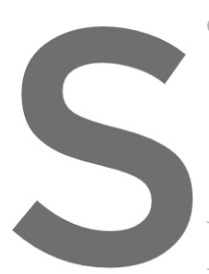
coordinates of each
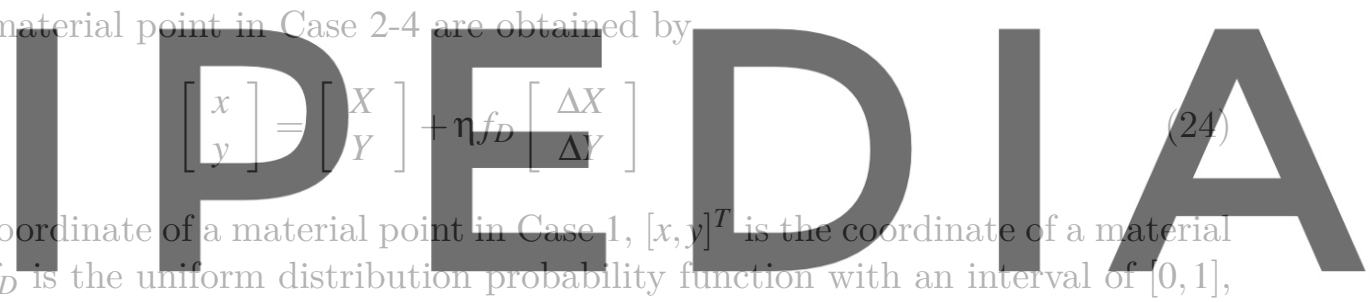

and $\eta$ is the scaling factor used to determine the randomness of the distribution of material

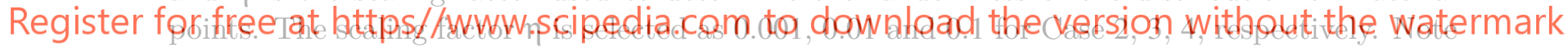

that when $\eta=0$, Cases 2-4 are degraded into the Case 1. Fig.7 shows the horizontal and vertical

displacement contours of material points for the four cases obtained by the proposed model. It can be seen from the figure that, similar displacement responses are observed for the four cases with different discretization, and zero-mode oscillation has been removed for each of the four cases.

\section{CONCLUSIONS}

- A stable Variational NOSB-PD model is proposed to unify the variational framework and boundary conditions of the NOSB-PD and the CCM.

- A force state vector formula in governing equations of the NOSB-PD is reformulated by introducing the first Piola-Kirchhoff stress.

- The proposed variational framework makes it easier to incorporate boundary conditions than standard peridynamics.

- A penalty method is introduced to eliminate the zero-energy mode oscillation inherently 


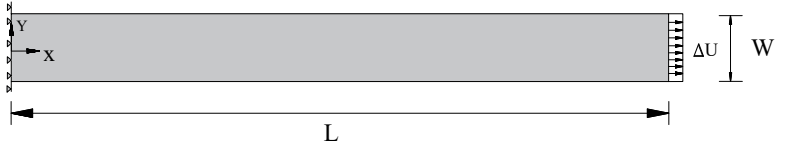

Figure 6: A hyper-elastic beam under uniaxial tension.

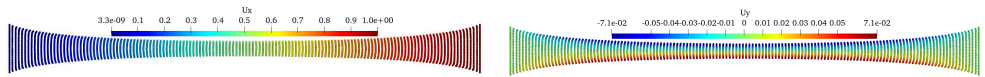

(a)
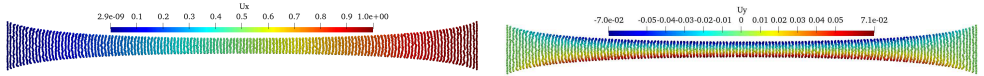

(b)
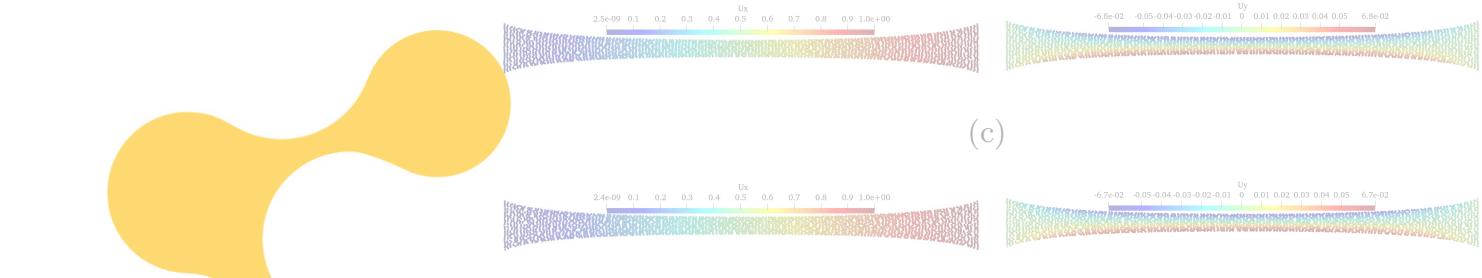

(c)

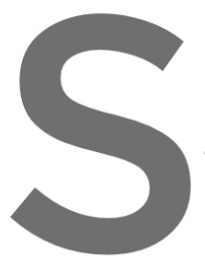

Figure 7 : The displaces
in $x$ and $y$ directions:
$\eta=0.1$. (unit: $m$ ).

observed in the NOSB-PD model.
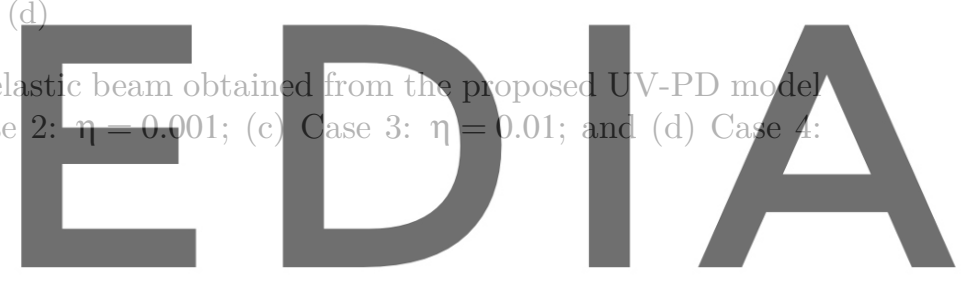

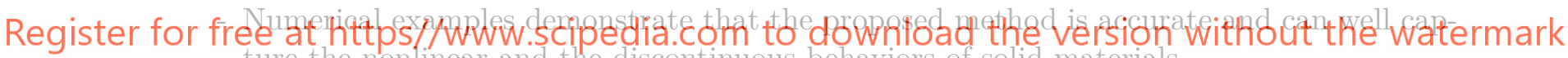
ture the nonlinear and the discontinuous behaviors of solid materials.

6 Acknowledgments

The research has been supported by the National Natural Science Foundation of China (41922059 \&51678438), the Shanghai Committee of Science and Technology (18DZ1205106), and the support from the Fundamental Research Funds for the Central Universities of China.

\section{REFERENCES}

[1] Belytschko, Ted and Black, Tom, Elastic crack growth in finite elements with minimal remeshing. Int. J. Num. Meth. Engng. (1999) 45:601-620.

[2] Belytschko, Ted and Lu, Yun Yun and Gu, Lei.Element-free Galerkin methods. Int. J. Num. Meth. Engng. (1994) 37:229-256.

[3] Sulsky, Deborah and Chen, Zhen and Schreyer, Howard L. A particle method for historydependent materials. Comput. Methods. Appl. Mech.Engng. (1994) 118:179-196. 
[4] Silling, Stewart A. Reformulation of elasticity theory for discontinuities and long-range forces. J. Mech. Phys. Solids. (2000) 48:175-209.

[5] Silling, Stewart A and Askari, Ebrahim. A meshfree method based on the peridynamic model of solid mechanics. Comput. Struct. (2005) 83:1526-1535.

[6] Gerstle, Walter and Sau, Nicolas and Silling, Stewart. Peridynamic modeling of concrete structures. "Nucl. Eng. Des. (2007) 237:1250-1258.

[7] Ren, Huilong and Zhuang, Xiaoying and Cai, Yongchang and Rabczuk, Timon.Dual-horizon peridynamics. Int. J. Num. Meth. Engng. (2016) 108:1451-1476.

[8] Zhu, Qi-zhi and Ni, Tao. Peridynamic formulations enriched with bond rotation effects.Int. J. Eng. Sci. (2017) 121:118-129.

[9] Diana, Vito and Casolo, Siro. A bond-based micropolar peridynamic model with shear deformability: Elasticity, failure properties and initial yield domains. Int. J. Solids. Struct. (2019) 160:201-231.

[10] Yu, Haitao and Chen, Xizhuo and Sun, Yuqi. A generalized bond-based peridynamic model for quasi-brittle materials enriched with bond tension-rotation-shear coupling effects. Comput. Methods. Appl. Mech.Engng. (2020) 372:113405.

[11] Mitchell, John Anthony. A Nonlocal Ordinary State-Based Plasticity Model for Peridynamics.Sandia National Lab.(SNL-NM), Albuquerque, NM (United States) (2011)

[12] Foster, John T and Silling, Stewart Andrew and Chen, Wayne W. Viscoplasticity using peridynamics. Int. J. Num. Meth. Engng. (2011) 81:1242-1258.

[13] Silling, Stewart A. Stability of peridynamic correspondence material models and their particle discretizations.Comput. Methods. Appl. Mech.Engng. (2017) 322:42-57.

[14] Silling, Stewart A and Lehoucq, Richard B. Peridynamic theory of solid mechanics. Adv. Appl. Mech. (2010) 44:73-168.

[15] Zhou, Xiao-Ping and Wang, Yun-Teng, Numerical simulation of crack propagation and coalescence in pre-cracked rock-like Brazilian disks using the non-ordinary state-based peridynamics. International Journal of Rock Mechanics and Mining Sciences (2016) 89:235-249.

[16] Nooru-Mohamed, MB and Schlangen, Erik and van Mier, Jan GM, Experimental and numerical study on the behavior of concrete subjected to biaxial tension and shear. Advanced cement based materials (1993) 1:22-37. 